\title{
The prevention and reduction of weight loss in an acute tertiary care setting: protocol for a pragmatic stepped wedge randomised cluster trial (the PRoWL project)
}

\author{
Alison L Kitson ${ }^{1,2^{*}}$, Timothy J Schultz ${ }^{1,3}$, Leslye Long ${ }^{1}$, Alison Shanks ${ }^{4}$, Rick Wiechula ${ }^{1}$, lan Chapman ${ }^{5}$ \\ and Stijn Soenen ${ }^{5}$
}

\begin{abstract}
Background: Malnutrition, with accompanying weight loss, is an unnecessary risk in hospitalised persons and often remains poorly recognised and managed. The study aims to evaluate a hospital-wide multifaceted intervention cofacilitated by clinical nurses and dietitians addressing the nutritional care of patients, particularly those at risk of malnutrition. Using the best available evidence on reducing and preventing unplanned weight loss, the intervention (introducing universal nutritional screening; the provision of oral nutritional supplements; and providing red trays and additional support for patients in need of feeding) will be introduced by local ward teams in a phased way in a large tertiary acute care hospital.
\end{abstract}

Methods/Design: A pragmatic stepped wedge randomised cluster trial with repeated cross section design will be conducted. The unit of randomisation is the ward, with allocation by a random numbers table. Four groups of wards ( $\mathrm{n}=6$ for three groups, $\mathrm{n}=7$ for one group) will be randomly allocated to each intervention time point over the trial. Two trained local facilitators (a nurse and dietitian for each group) will introduce the intervention. The primary outcome measure is change in patient's body weight, secondary patient outcomes are: length of stay, allcause mortality, discharge destinations, readmission rates and ED presentations. Patient outcomes will be measured on one ward per group, with 20 patients measured per ward per time period by an unblinded researcher. Including baseline, measurements will be conducted at five time periods. Staff perspectives on the context of care will be measured with the Alberta Context Tool.

Discussion: Unplanned and unwanted weight loss in hospital is common. Despite the evidence and growing concern about hospital nutrition there are very few evaluations of system-wide nutritional implementation programs. This project will test the implementation of a nutritional intervention across one hospital system using a staged approach, which will allow sequential rolling out of facilitation and project support. This project is one of the first evidence implementation projects to use the stepped wedge design in acute care and we will therefore be testing the appropriateness of the stepped wedge design to evaluate such interventions.

Trial registration: ACTRN12611000020987

Keywords: Malnutrition, Stepped wedge, Nutritional decline, Nutritional screening, MUST, Nutritional support, Oral nutritional supplement, Red tray, Evidence implementation, Knowledge translation, Feeding assistance, Nursing

\footnotetext{
* Correspondence: alison.kitson@adelaide.edu.au

${ }^{1}$ School of Nursing and Centre for Evidence-Based Practice SA (CEBSA),

University of Adelaide, Adelaide, SA 5005, Australia

${ }^{2}$ Green Templeton College, University of Oxford, Oxford, UK

Full list of author information is available at the end of the article
} 


\section{Background}

\section{Nutritional status of hospitalised patients}

The prevalence of malnutrition, defined here as proteinenergy under-nutrition causing measurable adverse effects on tissue/body form and function and clinical outcome [1] (p. S4), ranges from 20-60\% in older patients in Western acute healthcare [1-4]. Additionally, during hospitalisation nutritional status often declines in older patients due to a lack of adequate nutritional intake [5-7]. The adverse clinical outcomes associated with malnutrition and weight loss in acute care include increased: morbidity and mortality, length of stay, rates of infections, pressure sores and readmissions; and decreased functionality [1,8-14]. The economic costs of patient malnutrition and weight loss to healthcare are significant $[15,16]$.

In hospital settings, barriers to preventing nutritional decline have been shown to include a lack of staff knowledge and training [17], lack of prioritisation and timely feeding assistance by nursing staff [18], a lack of coordination between disciplines, including poor interdisciplinary communication, a lack of shared responsibility for nutrition care, and a lack of staff [19]. Naithani, Whelan et al. [20] reported that organisational, environmental and systems issues prevented malnourished patients, or those at risk of malnourishment, from adequately accessing food in the hospital setting. For example, patients were unable to open packaging, missed meals because of scheduled investigatory procedures, were not given food and fluids between mealtimes or these were placed out of reach, and lacked feeding assistance or were interrupted during mealtimes [20].

\section{Interventions to prevent nutritional decline in hospital patients}

The question of how to prevent weight loss and nutritional decline (these terms are used interchangeably in this protocol) in acute patients is complex. Interventions to improve patients' nutritional status and clinical outcomes have focussed on (i) the use of malnutrition screening tools, (ii) provision of nutritional support including supplements, and (iii) feeding assistance.

Nutritional screening in hospitals is required for accreditation in the US by the Joint Commission (http://www.jointcommission.org), is recommended in the UK by NICE [21] and the British Association for Parenteral and Enteral Nutrition (www.bapen.org.uk) and in Australia by the Dietitians Association of Australia as the first step in early detection of at risk patients [1,22]. Typically conducted by nurses [23], the evidence base for improved clinical benefits from screening, and subsequent timely and appropriate referral for nutrition care, is growing [24-26]. However, barriers to implementing nutrition screening tools in clinical practice are commonly encountered; screening rates in hospitals are typically only $60-70 \%$ and sometimes much lower [27-31]. A lack of time for what is considered a low priority is commonly cited by nursing staff as a barrier to nutritional screening of patients, additional reasons include lack of training, skills, knowledge and support, patients' short stays, and a need for enhanced collaboration between dietitians and nurses [27,29,30,32].

Of the large number of available nutritional screening tools [23], the Malnutrition Universal Screening Tool (MUST) developed in 2003 by the British Association for Parenteral and Enteral Nutrition [33] has been widely used [34-37]. The MUST has been shown to be quick (3-5 minutes) and accurate across a variety of hospital patient groups [26,37] and may be completed without weighing patients by using reliable patient or relative memory/recall $[26,28]$. However, the need for anthropometric measures and the presence of communication difficulties with confused patients are cited as limitations to using MUST [29,30]. Although some studies have claimed it 'easy' to use [37], other studies have reported difficulties in using the MUST [30,38].

Nutritional support aims to improve total energy and nutrient intake and may involve provision of oral nutritional supplements (ONS), or, if indicated, enteral tube feeding and/or parenteral nutrition [39]. There are a number of systematic reviews examining the effectiveness of ONS in improving nutritional status and clinical outcomes $[5,21,40,41]$. Although the review findings are somewhat inconsistent, the evidence suggests that supplementation produces small but consistent weight gain in older people and that improvements in mortality, morbidity (including complication rates) and functional status are greatest in underweight/undernourished patients $[5,40,41]$. The mean length of stay is not significantly reduced through ONS use [5].

The need for assisted eating is defined as "needing help from another person to be able to eat" ([42], p. 258). Assisted feeding ranges from verbal and non-verbal prompts to physical guiding to transferring food from the plate to an individual's mouth [43]. Up to $70 \%$ of elderly hospital patients require some feeding assistance [44], which is increasingly delivered by patient care assistants as opposed to nurses whose role at mealtimes and responsibilities for patient's nutritional status has diminished in recent years $[18,45,46]$. The use of a red tray to identify patients in need of feeding assistance has been advocated across the UK [47-49] and also implemented in a South Australian hospital. Although some studies have shown that nutritional intake can be improved [50,51], the evidence for the effectiveness of feeding assistance in improving patient outcomes is somewhat mixed [43,52,53].

Whilst there is a growing body of scientific evidence for single-faceted nutritional interventions in preventing nutritional decline and, in some cases, improving outcomes 
of older patients across a discrete sample of hospital wards, there is little research to guide or evaluate the effectiveness of multi-disciplinary, multi-faceted interventions implemented at an organisational level. The study outlined in this protocol aims to redress this imbalance and specifically sets out to address known barriers to implementing evidence about nutrition into clinical practice. Reflecting the focus on improving patient outcomes, the study is titled 'Prevention and Reduction of Weight Loss in Acute Care (PRoWL)'.

\section{Specific aims and hypotheses}

The study seeks to address four key questions. Firstly, does a multifaceted intervention incorporating a malnutrition screening tool, nutritional supplements and red trays implemented in a hospital improve outcomes for older, at risk patients? Secondly, does the intervention have any impact on staff-related factors (eg receptivity to evidence, staff behaviour and actions) and hospital processes related to nutrition? Thirdly, does the model of facilitated implementation of nutritional evidence support frontline staff to provide evidence-base care? Fourthly, what are the implications for implementing evidence on nutrition within an organisation in terms of successful strategies, sustainability, and study design?

The whole evaluation of the implementation, which will include a stepped wedge design and a full process evaluation of facilitator and staff experiences, will add to existing strategies for implementing nutritional evidence into practice, thereby addressing questions 3 and 4 . The following hypotheses that are most pertinent to this study protocol relate to questions 1 and 2 only:

1.1The multifaceted nutrition intervention will improve outcomes (e.g. prevent nutritional decline, reduce mortality, readmission rates) for older, at risk patients.

2.1The multifaceted nutrition intervention will improve staff-related outcomes (e.g. receptivity to evidence, staff behaviour).

2.2The multifaceted nutrition intervention will improve hospital processes related to patient nutrition (e.g. weighing patients, screening patients, documentation).

\section{Methods/Design}

\section{The intervention}

The evidence-based intervention will consist of three linked activities: the introduction and use of the Malnutrition Universal Screening Tool (MUST), the provision of food supplements to patients identified at risk of malnutrition, and the introduction of a system that uses red feeding trays to flag patients requiring full feeding assistance. A multidisciplinary partnership between implementation science researchers (AK, RW, TS), clinicians (AS, LL) and nutrition researchers (SS, IC) was formed to assist in facilitating and evaluating the intervention.

\section{The implementation}

The intervention will be introduced in a staged way across an entire hospital using a stepped wedge design $[54,55]$ described in more detail in the next section. The implementation of the intervention will be facilitated by a nurse paired with a hospital dietitian. The four nurse leads will be identified prior to the project commencing and their wards will be allocated to one of the four groups using stratified randomisation. For three of the four groups an additional five hospital wards will be randomly assigned to the group; the last group will be assigned an additional six wards meaning that a total of 25 wards are allocated (Table 1). Allocation of wards to groups will be conducted (by author TS) by coding each ward and using a random number generator (MS-Excel) to select codes for each groups. The intervention will be implemented over a two month period across all six wards within a group but staggered sequentially every three months between groups. Control sites will experience usual hospital care until the intervention is delivered. The intervention will be carried out over 12 months, commencing in March 2011.

The facilitating nurse-dietitian pairs will be trained in clinical leadership, evidence translation and the intervention (nutritional screening, supplements and the use of red trays prior to the implementation). The structured training available to facilitators will include: a short fellowship at the Joanna Briggs Institute (www.joannabriggs.edu.au) to develop evidence implementation and clinical leadership skills, the use of a tool kit containing materials to support their education and leadership roles, and a training pack of clinical content to deliver education to nurses working in their wards. The training materials will be jointly developed with, and facilitators will be supported by, two members of the research team (AK and RW). The clinical content will be developed prior to the roll out by the facilitators working with a dietitian (AS).

\begin{tabular}{|c|c|c|c|c|c|c|}
\hline & & \multicolumn{5}{|c|}{ Time period } \\
\hline & & 1 & 2 & 3 & 4 & 5 \\
\hline \multirow[t]{4}{*}{ Group/Step } & 1 (six wards) & 0 & $x$ & $x$ & $x$ & X \\
\hline & 2 (six wards) & 0 & 0 & $x$ & $x$ & $x$ \\
\hline & 3 (six wards) & 0 & 0 & 0 & $x$ & $x$ \\
\hline & 4 (seven wards) & 0 & 0 & 0 & 0 & $x$ \\
\hline
\end{tabular}

In this trial, 25 hospital wards are randomised to four groups-three groups of six wards and one group of seven wards. 
The role of the facilitator pairs will be to identify 'champions' on each ward and to work with champions to support the roll out of all three components of the intervention before, during and after the intervention phase. The facilitator pairs will train the champions to deliver education and work with champions to undertake unit level audits to determine local compliance with key nutritional evidence-based clinical processes before and after the intervention is implemented, and will assist champions to feed back these local data at each ward.

\section{Stepped wedge trial}

Though infrequently used and poorly known, awareness and use of the stepped wedge trial design is increasing [54,56-58]. Stepped wedge trials are a type of crossover trial in which different groups (or steps) cross over (ie switch from being a control to a treatment) at different time points [59]. In comparison to other crossover trials, the groups/steps only cross in one direction, from control to treatment. The first time point (Time 1) is a baseline measurement, and groups/steps are then randomised to receive the intervention at subsequent time points (Table 1) until all groups/steps have received the intervention [59]. Data is collected from all groups at regular intervals during the study, including at baseline and whenever a new group receives the intervention. At the conclusion of the trial, each of the units in each group have been exposed to the intervention, and each of the groups has a varying amount of control data collected prior to receiving the intervention (Table 1).

A stepped wedge randomised cluster trial with repeated cross section design will be employed $[54,55,59]$. The 'step' refers to each group of wards; and the 'cluster' refers to the ward as unit of randomisation. The 'repeated cross section' measures refer to the fact that multiple cross sectional measures are made with different patients in each cluster for each of the sampling time periods [55], as recently used in surgical patients across three hospitals [58].

The stepped wedge allows all clusters to receive the intervention. Further, unlike a conventional crossover trial, no clusters are removed from the intervention, which is ethically preferable if there is the prior belief that the intervention should result in benefits for the patient population $[54,55,59]$. The design also allows for a staged implementation, which is particularly beneficial if there are logistical, practical or financial limitations to rolling out an intervention at all sites in one time period [59]. The design supports evidence implementation in complex, changeable environments because the staged implementation allows improvements to be made to the intervention, for example training materials can be updated to accommodate feedback from participants. Analysis of stepped wedge trials can adjust for possible temporal trends in the effectiveness of the intervention, and the design reduces the impact of intra-cluster correlations on power because each group acts as its own control [54]. A disadvantage of the design is the need for data collection from all units at each sampling period, and potential lengthening of the trial to allow sequential roll out of the intervention across all groups [54]. These attributes support the use of routinely collected hospital administrative data as outcomes of stepped wedge trials.

\section{The setting}

The study will be conducted in a large tertiary South Australian acute care hospital in which patient care areas are divided into six divisions and a total of 26 wards/units. A recent evidence implementation project on nutrition screening and documentation found substantial improvement from low baseline levels in two hospital wards and identified more widespread interest for further improvement to nutritional status and care of vulnerable patients [60,61]. Additionally, a number of internal, unpublished audits have identified that up to half of patients are at risk of nutritional decline in a system that does not prioritise patient nutrition.

\section{Inclusion and exclusion criteria}

Critical care areas will be excluded from the study, all remaining 25 wards will be allocated to one of the four stepped wedge groups. Otherwise, there are no exclusion criteria for implementing the intervention-all nursing staff and patients on the wards will be eligible to be exposed to the intervention. The patients will all be over 18 years of age with no upper age limitation.

\section{Outcomes}

The primary patient outcome measure will be the rate of change in body mass and body mass index over weekly periods from admission to discharge. Body mass $(\mathrm{kg})$ will be measured to the nearest $0.1 \mathrm{~kg}$, between 0800 and 1200 and post bladder voiding. Depending on patient mobility this may require a standing, sitting, bed or sling weighing machine. Patient's height $(\mathrm{cm})$ will be measured to the nearest $0.1 \mathrm{~cm}$ using a stadiometer or predicted from measured ulna length depending on patient mobility. Body mass index $\left(\mathrm{kg} / \mathrm{m}^{2}\right)$ is calculated as body weight divided by height squared. The body mass data will be collected by a researcher (LL), who is not blinded to the randomisation schedule.

Secondary patient outcome measures will include: length of stay, all cause mortality, discharge destination (to a higher, lower or equivalent level of care), number and duration of re-admissions (defined as an unplanned admission within 28 days of discharge related to the primary admission [62] (3, 6, 12 months post-discharge), and number of Emergency Department (ED) presentations (3, 6, 
12 months post-discharge). The re-admission and ED presentations data will be collected from across all five major public hospitals in the metropolitan region using the State's patient record systems, which will also provide length of stay, discharge destination and mortality, crossreferenced with State death registries. The collector of the secondary patient outcome data will be blinded to the randomisation schedule.

The staff-related outcome is nurse perspectives of context of care as assessed by administration of the Alberta Context Tool (ACT) $[63,64]$ before the intervention.

Hospital process-related outcomes include: compliance to using the MUST tool on all patients, compliance to using additional food supplements when indicated, compliance to using the red tray system when indicated and compliance to documenting patient's foods and fluids intake by nurses. The hospital process measures will be obtained through medical record review.

\section{Sampling}

The primary outcome data will be collected on a sample of patients from only one ward per group due to resource constraints. Given that the median length of stay in the hospital is only 4-5 days, and our requirement is to collect weight data over at least a 1 week period, we will purposively sample a ward that is most likely to care for patients who have a length of stay in excess of 7 days. To further minimise the number of patients for whom a weight on admission is collected but who are discharged before 7 days, patients with Diagnostic Related Groups (DRGs) known to have longer than average length of stay will be targeted. The following patients will be excluded from body mass measurement: those undergoing palliation or expected to die within a month, those undergoing amputation or surgical removal of large tumours, those admitted directly to the intensive care unit or with stays in intensive care of more than 3 weeks. A total of 20 patients for each of four wards will be weighed on each of five measurement periods, resulting in a total sample size of 400. Whilst it was desirable to include either more wards or more patients per ward (see power calculations in Analysis) in the sample, resource constraints limited the final sample size available for analysis. Given the size of the wards (typically 20-24 patients each) it is anticipated that it may take repeated weekly visits over an entire month to reach a total sample of 20 patients with body weight measurements for each sampling period. Patients whose length of stay exceeds 14 days will be weighed at day 14 and day 21 to allow calculation of change in weight over the first, second and third weeks of their admission. The second and third week data will both be analysed separately.

Secondary outcomes and hospital process measures will only be collected from the records of patients included in the primary outcome sample, described above. Written consent to weigh patients will not be sought because it is routine practice and part of usual care in the hospital.

\section{Analysis}

Analysis of stepped wedge designs is challenging [54]. The unidirectional aspect of stepped wedged trials complicates analysis because the treatment effect cannot be estimated exclusively from within-cluster comparisons [59]. Analytical techniques depend on a number of factors including: the existence of a temporal effect on the outcome, equality or inequality in cluster size, and normality of continuous data. Based on a design using equal cluster sizes, and assuming that there will be a temporal effect, linear mixed models (LMM) will be used [65]. If there are no temporal effects, then within-cluster analysis can be used to estimate the treatment effect. Random effects will be used to model correlation between individuals within the same cluster and measure the variance of individuals in a cluster and variance at the cluster-level [59]. Hussey et al. (2007) provide equations and worked examples for calculating both the within-cluster and between-cluster calculations [59].

We conducted power analysis based on a sub-set of body weight data collected at baseline at four hospital wards. In their first week of admission, the prevalence of patients with (i) BMI greater than or equal to 20 who lost 2 or more $\mathrm{kg}$, and (ii) BMI less than 20 who lost $1 \mathrm{~kg}$ or more, was measured as $26.7 \%$. Using equations seven and eight [59], a coefficient of variation $\left(\frac{\tau}{\mu}=\frac{0.056}{0.267}\right)$ of 0.21 , with four groups of 20 patients measured over five time periods totalling 400 patients, a power of $80 \%$ would detect a reduction in the prevalence of weight loss from $26.7 \%$ to $6.5 \%$, ie a $75 \%$ reduction. If a smaller effect size is selected, such as a reduction in prevalence from $26.7 \%$ to $16.5 \%$ (a $38 \%$ reduction), the power is reduced to $29 \%$.

It is also possible to calculate the required sample size for an adequately (80\%) powered study. To detect a smaller effect size (a 38\% reduction in proportion of patients experiencing weight loss), the total sample size required is 1800 patients, comprising 90 patients in each of four groups measured across the five time periods. Alternatively, although not feasible for reasons defined above, if all 25 wards were included in the sample and 20 patients were included from each ward at each of five time periods, the resulting study comprising a total of 2,500 patients would have a power of $69 \%$ to detect a $38 \%$ reduction in proportion of patients experiencing weight loss.

All of the outcomes except for the Alberta Context Tool will be analysed according to the stepped wedge design, as these data are all collected at each of the five measurement time periods of the study. The Alberta 
Context Tool will be compared using an hierarchical (nested) ANOVA to account for variation within, and between, wards. Analysis will be conducted using Stata software.

\section{Ethics}

The study was approved by the University of Adelaide Human Research Ethics and the hospital's Ethics Committee.

\section{Discussion}

The vulnerability of older adults to nutritional decline may be both a cause, and an effect, of illness [66]. Therefore, preventing nutritional decline is neither possible nor a realistic goal for all hospitalised, older patients and the difficulties in improving patient outcomes in through nutrition interventions are well known $[53,67]$. Although nutrition has been consistently defined over the last 150 years in the literature and in practice as one of the core nursing fundamentals of care [68], the evidence suggests that multidisciplinary approaches are most likely to prevent nutritional decline [69], particularly as responsibility for nutrition has diffused between multiple disciplines (e.g. nursing, medicine, dietetics and other allied health such as speech pathology and occupational therapy) [6]. Therefore we have proposed an intervention and developed a research team that is both multidisciplinary and team-led. We believe that the interdisciplinary partnerships built into the intervention and study design will lead to a stronger intervention that is more likely to be successful in the short term and sustainable in the long term. Although medical input into the implementation has been provided via the research team, it is unclear how further engagement with doctors around the importance of nutrition in practice will be obtained.

The multifaceted intervention developed for this project is novel, bringing together evidence-based elements for which local support also existed. Additionally, the intervention has sought to address some of the key factors impacting nutritional intake of older patients, particularly those that make it easier for staff to be aware that a patient is at risk of nutritional decline [20]. The use of MUST as the screening tool in this study reflects previous evidence implementation work conducted in this setting [61]. While there is no 'gold standard' for malnutrition screening, apart from familiarity, reasons for selecting MUST in this study include that it: allows for screening and establishing nutritional risk, has been widely used, and has good reliability and validity [70].

The implementation designed for this project is based on the PARIHS framework, which proposes that successful evidence implementation is a function of the evidence, the context and the facilitation [71,72]. To best engage with end-users of the evidence (frontline clinical staff) we have built in two levels of facilitation in the implementation (between nurse-dietitian pairs and wards, and between the research team and the nurse-dietitian pairs). Further we have proposed a novel application of the Alberta Context Tool. As part of the intervention, the tool will be used to assess existing context and as the basis for feedback to wards on their perceived strengths and weaknesses.

Our preliminary work towards completing the proposed study has already identified a number of barriers to implementing the intervention into practice. In particular, we have identified the need for each ward to have access to accurate and appropriate scales (e.g. weigh chairs and beds) with which to weigh patients on admission and weekly thereafter, and the difficulties of adding an additional form (the MUST) to the patient record.

We have selected the stepped wedge trial as an innovative approach to a cluster randomised crossover trial [59] subject to the constraints of (i) limited resources to roll out the intervention across the entire hospital, (ii) a belief that the intervention will do more good than harm, therefore should not be withheld from any part of the hospital [57], and (iii) a desire to be able to flexibly modify aspects of the intervention as befitting a realist evaluation [73]. In this instance, the evaluation of a multifaceted intervention that has evidence for its individual components is, in theory, similar to the indicated use of stepped wedge trials where "interventions likely to do more good than harm, a stepped wedge design may be particularly beneficial in evaluating interventions being implemented in a new setting, where evidence for their effectiveness in the original setting is available, or for patient safety interventions that have undergone careful pre-implementation evaluation to rule out any collateral damage" [55] (p. 9).

The study design and analysis are, however, complicated and novel. Review of CONSORT statements for reporting of randomised controlled trials (e.g. [74]) indicates that this design includes a number of non-standard elements that impact the design and reporting of the study. For example, the study is a pragmatic trial, in that it seeks to establish the effectiveness of the intervention in normal practice using a flexibly implemented intervention to directly inform policy and "real world choices" [75]. Secondly, the study assesses a nonpharmacologic intervention, with accompanying challenges around the complexity of the intervention, the expertise of care providers and difficulties in blinding [76,77]. Thirdly, the study uses clustering, which has particular implications for design and analysis, blinding, and recruitment of patients within a cluster [78], and study power [59]. Meeting the individual reporting guidance for each of the three nonstandard elements to this study (a pragmatic, cluster randomised trial of nonpharmaceutical interventions) 
represents a substantial effort to be addressed in the final reporting of the study $[75,76,78]$.

The decision to focus on a single facility in this study is a potential limitation but reflects a number of constraints. Firstly, our work assessing context in this facility has shown substantial variation between wards in many dimensions, including leadership, culture and feedback processes [64]. Therefore, we tailored an intervention that involved ward-based champions to address local contextual factors and assist in the collection and feeding back of local audit data collected at the ward level. Secondly, although based on research evidence, the intervention developed for this project was novel and best piloted at a single site before further testing at a health system-wide level (i.e. multiple organisation involvement) is conducted. Lastly, the limited resources available were most appropriately used by concentrating on a single facility. Another limitation to this study, also related to limited resources, is the absence of biochemical markers of metabolic status, such as transthyretin and albumin [79].

Finally, although the project was originally envisaged as accessing routinely collected [54] patient body mass data, initial investigation of this dataset indicated systematic biases and large gaps rendering it unfit for this purpose. Therefore we decided to prospectively collect body mass data on a targeted sub-section of the population. This approach has introduced limitations related to power, as the study will be under-powered to detect anything but large effect sizes, and in generalisability, as we will purposively sample from the patient population and target those with a length of stay likely to be at least 1 week. However, as we are anticipating the results from this study to be preliminary in nature, and to potentially inform a larger study, this was necessary as the first step towards investigating the multifaceted intervention. Further embedding of nutrition screening and weighing of patients will provide a data source for future studies evaluating long term sustainability of the intervention.

\section{Conclusion}

We have outlined a study that we anticipate will contribute to the knowledge base of nutritional care of patients in hospitals and evidence translation across multidisciplinary healthcare. The pragmatic stepped wedge randomised trial offers a novel solution to implementation science in healthcare, particularly when resources are limited, and/or there is a prior belief that the intervention will do more good than harm.

\section{Abbreviations}

ED: Emergency department; ACT: Alberta Context Tool; UK: United Kingdom; US: United States; MUST: Malnutrition Universal Screening Tool; DRGs: Diagnostic Related Groups; BMI: Body Mass Index.

\section{Competing interests}

The authors declare that they have no competing interests.

\section{Authors' contributions}

AK: conceived the study (with LL), drafted the first versions of the protocol, brought the research team together, led the project development, chaired the research steering group and will support facilitator pairs with RW.

TS: conducted literature review, developed and refined the study design and analysis, and will manage the context assessment. LL: conceived the study (with AK), identified appropriate outcomes and assisted in the implementation plan. AS: developed the dietetic component to the intervention and led dietetic and catering aspects to the implementation, identified appropriate study outcomes. RW: developed the training materials with facilitators and will support facilitator pairs with AK. IC: identified appropriate and clinically sensitive outcomes and assisted with study design. SS: identified appropriate and clinically sensitive outcomes and assisted with study design. All authors were members of the project steering group, and contributed to drafts of the protocol. All authors read and approved the final manuscript.

\section{Acknowledgements}

The project has been part funded by the Royal Adelaide Hospital Nursing Education Fund. Nancy Whitaker (University of Adelaide) assisted in compiling training materials and administering the questionnaires. Statistical advice was provided by Tom Sullivan (University of Adelaide). Gail Whitelock and Mark Ramage developed the clinical tool kit for facilitator pairs.

Di Liebelt facilitated access to hospital records systems.

\section{Author details}

${ }^{1}$ School of Nursing and Centre for Evidence-Based Practice SA (CEBSA), University of Adelaide, Adelaide, SA 5005, Australia. ${ }^{2}$ Green Templeton College, University of Oxford, Oxford, UK. ${ }^{3}$ Australian Patient Safety Foundation, University of South Australia, Mailbox CEA-20, Adelaide, SA 5000, Australia. ${ }^{4}$ Clinical Dietetics, Royal Adelaide Hospital, North Terrace, Adelaide, SA 5000, Australia. ${ }^{5}$ Centre of Research Excellence (CRE) in Translating Nutritional Science to Good Health, School of Medicine, University of Adelaide, Adelaide, SA 5005, Australia.

Received: 29 March 2012 Accepted: 5 August 2013

Published: 8 August 2013

\section{References}

1. Watterson C, Fraser A, Banks M, Isenring E, Miller M, Silvester C, Hoevenaars $R$, Bauer J, Vivanti A, Ferguson M: Evidence based practice guidelines for the nutritional management of malnutrition in adult patients across the continuum of care. Nutr Diet 2009, 66:S1-S34.

2. McWhirter JP, Pennington CR: Incidence and recognition of malnutrition in hospital. Br Med J 1994, 308:945-948.

3. Middleton MH, Nazarenko G, Nivison-Smith I, Smerdely P: Prevalence of malnutrition and 12-month incidence of mortality in two Sydney teaching hospitals. Intern Med J 2001, 31:455-461.

4. Thibault R, Chikhi M, Clerc A, Darmon P, Chopard P, Genton L, Kossovsky MP, Pichard C: Assessment of food intake in hospitalised patients: a 10year comparative study of a prospective hospital survey. Clin Nutr 2011, 30:289-296.

5. Milne AC, Potter J, Vivanti A, Avenell A: Protein and energy supplementation in elderly people at risk from malnutrition. Cochrane Database Syst Rev 2009. Issue 2. Art. No.: CD003288. http://onlinelibrary. wiley.com/doi/10.1002/14651858.CD003288.pub3/pdf.

6. Mudge AM, Ross $\sqcup$, Young AM, Isenring EA, Banks MD: Helping understand nutritional gaps in the elderly (HUNGER): a prospective study of patient factors associated with inadequate nutritional intake in older medical inpatients. Clin Nutr 2011, 30:320-325.

7. Sullivan DH, Sun S, Walls RC: Protein-energy undernutrition among elderly hospitalized patients: a prospective study. JAMA 1999, 281:2013-2019.

8. Covinsky KE, Martin GE, Beyth RJ, Justice AC, Sehgal AR, Landefeld CS: The relationship between clinical assessments of nutritional status and adverse outcomes in older hospitalized medical patients. J Am Geriatr Soc 1999, 47:532-538.

9. Kagansky N, Berner Y, Koren-Morag N, Perelman L, Knobler H, Levy S: Poor nutritional habits are predictors of poor outcome in very old hospitalized patients. Am J Clin Nutr 2005, 82:784-791. quiz 913-784.

10. Klein S, Kinney J, Jeejeebhoy K, Alpers D, Hellerstein M, Murray M, Twomey $P$, Bistrian B, Bothe A, Heitkemper M, et al: Nutrition support in clinical 
practice: review of published data and recommendations for future research directions. J Parenter Enteral Nutr 1997, 21:133-156.

11. Larsson J: Clinical problem of malnutrition. Nutrition 1993, 9:274-275.

12. Mudge AM, Kasper K, Clair A, Redfern H, Bell JJ, Barras MA, Dip G, Pachana NA: Recurrent readmissions in medical patients: a prospective study. J Hosp Med 2011, 6:61-67.

13. Schneider SM, Veyres $P$, Pivot X, Soummer AM, Jambou P, Filippi J, van Obberghen $E$, Hébuterne $X$ : Malnutrition is an independent factor associated with nosocomial infections. Br J Nutr 2004, 92:105-111.

14. Sullivan $\mathrm{DH}$ : Risk factors for early hospital readmission in a select population of geriatric rehabilitation patients: the significance of nutritional status. J Am Geriatr Soc 1992, 40:792-798.

15. Amaral TF, Matos LC, Tavares MM, Subtil A, Martins R, Nazaré M, Sousa Pereira N: The economic impact of disease-related malnutrition at hospital admission. Clin Nutr 2007, 26:778-784.

16. Russell CA: The impact of malnutrition on healthcare costs and economic considerations for the use of oral nutritional supplements. Clin Nutr Supp/ 2007, 2:25-32

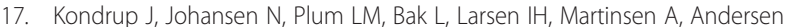
$J R$, Bærnthsen $H$, Bunch $E$, Lauesen $N$ : Incidence of nutritional risk and causes of inadequate nutritional care in hospitals. Clin Nutr 2002, 21:461-468

18. Xia C, McCutcheon H: Mealtimes in hospital-who does what? J Clin Nurs 2006, 15:1221-1227.

19. Ross L, Mudge AM, Young AM, Banks M: Everyone's problem but nobody's job: staff perceptions and explanations for poor nutritional intake in older medical patients. Nutr Diet 2011, 68:41-46.

20. Naithani S, Whelan K, Thomas J, Gulliford MC, Morgan M: Hospital inpatients' experiences of access to food: a qualitative interview and observational study. Health Expect 2008, 11:294-303.

21. National Institute for Health and Clinical Excellence: Nutrition support in adults: oral nutrition support, enteral tube feeding and parenteral nutrition. Clinical guideline 32. London: National Institute for Health and Clinical Excellence (NICE); 2006

22. Watterson C, Miller M, Ferguson M, Isenring E, Bauer J, Silvester C, Fraser A, Banks M, Vivanti A, Hoevenaars R: Beware: screening is not assessment when it comes to malnutrition. Nutr Diet 2010, 67:122-123.

23. Green SM, Watson R: Nutritional screening and assessment tools for older adults: literature review. J Adv Nurs 2006, 54:477-490.

24. Elia M, Zellipour L, Stratton RJ: To screen or not to screen for adult malnutrition? Clin Nutr 2005, 24:867-884.

25. Kruizenga HM, Van Tulder MW, Seidell JC, Thijs A, Ader HJ, Van Bokhorst-de van der Schueren MA: Effectiveness and cost-effectiveness of early screening and treatment of malnourished patients. Am J Clin Nutr 2005, 82:1082-1089.

26. Stratton RJ, King CL, Stroud MA, Jackson AA, Elia M: 'Malnutrition Universal Screening Tool' predicts mortality and length of hospital stay in acutely ill elderly. Br J Nutr 2006, 95:325-330

27. Bailey R: Implementing nutrition screening. Nurs Manage 2006, 13:20-24

28. Lamb CA, Parr J, Lamb EIM, Warren MD: Adult malnutrition screening prevalence and management in a United Kingdom hospital: crosssectional study. Br J Nutr 2009, 102:571-575.

29. Porter J, Raja R, Cant R, Aroni R: Exploring issues influencing the use of the malnutrition universal screening tool by nurses in two Australian hospitals. J Hum Nutr Diet 2009, 22:203-209.

30. Raja R, Gibson S, Turner A, Winderlich J, Porter J, Cant R, Aroni R: Nurses' views and practices regarding use of validated nutrition screening tools. Aust J Adv Nurs 2008, 26:26-33.

31. Wong S, Gandy J: An audit to evaluate the effect of staff training on the use of Malnutrition Universal Screening Tool. J Hum Nutr Diet 2008, 21:405-406.

32. Villalon L, Laporte M, Carrier N: Nutrition screening for seniors in health care facilities: a survey of health professionals. Can J Diet Pract Res 2011, 72:162-169.

33. Elia M: Screening for malnutrition: a multidisciplinary responsibility development and use of the Malnutrition Universal Screening Tool ('MUST') for adults. BAPEN: Redditch; 2003.

34. Crosbie J, Jaafar A, Yuill S, Hawkyard C, Lapworth K, Davidson B, Thompson NP: Assessing the impact of the Malnutrition Universal Screening Tool (MUST) in identifying malnourished patients. CME Journal Gastroenterology, Hepatology and Nutrition 2008, 9:66-69.
35. Jaafar A, Hawkyard C, Lapworth K, Davidson B, Ledger J, Wilkins J, Mansfield J, Thompson NP: Screening for malnutrition: impact of the malnutrition universal screening tool (MUST). Gut 2003, 52:A11-A11.

36. Parsons E: Nutritional care and the Malnutrition Universal Screening Tool (MUST). Br J Community Nurs 2011, 16:S16-S21.

37. Stratton RJ, Hackston A, Longmore D, Dixon R, Price S, Stroud M, King C, Elia M: Malnutrition in hospital outpatients and inpatients: prevalence, concurrent validity and ease of use of the 'Malnutrition Universal Screening Tool' ('MUST') for adults. Br J Nutr 2004, 92:799-808.

38. Neelemaat F, Meijers J, Kruizenga H, Van Ballegooijen H, Van Bokhorst-de van der Schueren M: Comparison of five malnutrition screening tools in one hospital inpatient sample. J Clin Nurs 2011, 20:2144-2152.

39. Stratton RJ, Elia M: Who benefits from nutritional support: what is the evidence? Eur J Gastroenterol Hepatol 2007, 19:353-358.

40. Stratton RJ, Elia M: A review of reviews: a new look at the evidence for oral nutritional supplements in clinical practice. Clin Nutr Supp/ 2007 2:5-23.

41. Vanderkroft D, Collins CE, Fitzgerald M, Lewis S, Neve M, Capra S: Minimising undernutrition in the older inpatient. Int J Evid Based Healthc 2007, 5:110-181

42. Westergren A, Karlsson S, Andersson P, Ohlsson O, Hallberg IR: Eating difficulties, need for assisted eating, nutritional status and pressure ulcers in patients admitted for stroke rehabilitation. J Clin Nurs 2001 10:257-267.

43. Green SM, Martin HJ, Roberts HC, Sayer AA: A systematic review of the use of volunteers to improve mealtime care of adult patients or residents in institutional settings. J Clin Nurs 2011, 20:1810-1823.

44. Tsang MF: Is there adequate feeding assistance for the hospitalised elderly who are unable to feed themselves? Nutrition \& Dietetics 2008, 65:222-228.

45. Dickinson A, Welch C, Ager L: No longer hungry in hospital: improving the hospital mealtime experience for older people through action research. J Clin Nurs 2008, 17:1492-1502.

46. Jefferies $D$, Johnson $M$, Ravens J: Nurturing and nourishing: The nurses' role in nutritional care. J Clin Nurs 2011, 20:317-330.

47. Age UK: Still hungry to be heard. London: Age UK; 2010.

48. Bradley $L$, Rees $C$ : Reducing nutritional risk in hospital: the red tray. Nurs Stand 2003, 17:33-37.

49. Davis C: Mealtime solutions. Nurs Stand 2007, 21:21-23.

50. Walton K, Williams P, Bracks J, Zhang QS, Pond L, Smoothy R, Tapsell L, Batterham $M$, Vari $L$ : A volunteer feeding intakes of assistance program can improve dietary elderly patients-a pilot study. Appetite 2008 , 51:244-248

51. Wright $L$, Cotter D, Hickson M: The effectiveness of targeted feeding assistance to improve the nutritional intake of elderly dysphagic patients in hospital. J Hum Nutr Diet 2008, 21:555-562.

52. Duncan DG, Beck SJ, Hood K, Johansen A: Using dietetic assistants to improve the outcome of hip fracture: a randomised controlled trial of nutritional support in an acute trauma ward. Age Ageing 2006, 35:148-153.

53. Hickson M, Bulpitt C, Nunes M, Peters R, Cooke J, Nicholl C, Frost G: Does additional feeding support provided by health care assistants improve nutritional status and outcome in acutely ill older in-patients?-a randomised control trial. Clin Nutr 2004, 23:69-77.

54. Brown C, Hofer T, Johal A, Thomson R, Nicholl J, Franklin BD, Lilford RJ: An epistemology of patient safety research: a framework for study design and interpretation. Part 2. Study design. Qual Saf Health Care 2008, 17:163-169.

55. Brown CA, Lilford RJ: The stepped wedge trial design: a systematic review. BMC Med Res Methodol 2006, 6:54

56. Liddy C, Hogg W, Russell G, Wells G, Armstrong CD, Akbari A, Dahrouge S, Taljaard M, Mayo-Bruinsma L, Singh J, Cornett A: Improved delivery of cardiovascular care (IDOCC) through outreach facilitation: study protocol and implementation details of a cluster randomized controlled trial in primary care. Implementation Science 2011, 6:110.

57. Mdege ND, Man MS, Taylor CA, Torgerson DJ: Systematic review of stepped wedge cluster randomized trials shows that design is particularly used to evaluate interventions during routine implementation. J Clin Epidemiol 2011, 64:936-948.

58. Mouchoux C, Rippert P, Duclos A, Fassier T, Bonnefoy M, Comte B, Heitz D Colin C, Krolak-Salmon P: Impact of a multifaceted program to prevent 
postoperative delirium in the elderly: the CONFUCIUS stepped wedge protocol. BMC Geriatr 2011, 11:25.

59. Hussey MA, Hughes JP: Design and analysis of stepped wedge cluster randomized trials. Contemp Clin Trials 2007, 28:182-191.

60. Kitson A, Silverston H, Wiechula R, Zeitz K, Marcoionni D, Page T: Clinical nursing leaders', team members' and service managers' experiences of implementing evidence at a local level. J Nurs Manag 2011, 19:542-555.

61. Wiechula R, Kitson A, Marcoionni D, Page T, Zeitz K, Silverston H: Improving the fundamentals of care for older people in the acute hospital setting: facilitating practice improvement using a Knowledge Translation Toolkit. Int J Evid Based Healthc 2009, 7:283-295.

62. Australian Council on Healthcare Standards: Australasian clinical indicator report: 2001-2009. Determining the potential to improve quality of care. 11th edition. Sydney: Australian Council on Healthcare Standards; 2010.

63. Estabrooks CA, Squires JE, Cummings GG, Birdsell JM, Norton PG: Development and assessment of the Alberta Context Tool. BMC Health Serv Res 2009, 9:234

64. Schultz TJ, Kitson AL: Measuring the context of care in an Australian acute care hospital: a nurse survey. Implementation Science 2010, 5:60.

65. Laird N, Ware J: Random-effects models for longitudinal data. Biometrics 1982, 38:963-974.

66. Holmes S: Nutrition and eating difficulties in hospitalised older adults. Nurs Stand 2008, 22:47-57. quiz 58, 60 .

67. Doig GS, Simpson F, Finfer S, Delaney A, Davies AR, Mitchell I, Dobb G, Grp ACT: Effect of evidence-based feeding guidelines on mortality of critically ill adults a cluster randomized controlled trial. JAMA 2008, 300:2731-2741.

68. Kitson A, Conroy T, Wengstrom Y, Profetto-McGrath J, Robertson-Malt S: Defining the fundamentals of care. Int J Nurs Pract 2010, 16:423-434.

69. Hoekstra JC, Goosen JHM, de Wolf GS, Verheyen CCPM: Effectiveness of multidisciplinary nutritional care on nutritional intake, nutritional status and quality of life in patients with hip fractures: a controlled prospective cohort study. Clin Nutr 2011, 30:455-461.

70. Elia M, Stratton RJ: Considerations for screening tool selection and role of predictive and concurrent validity. Curr Opin Clin Nutr Metab Care 2011 14:425-433.

71. Kitson A, Harvey G, McCormack B: Enabling the implementation of evidence based practice: a conceptual framework. Qual Health Care 1998, 7:149-158.

72. Kitson AL, Rycroft-Malone J, Harvey G, McCormack B, Seers K, Titchen A: Evaluating the successful implementation of evidence into practice using the PARiHS framework: theoretical and practical challenges. Implementation Science 2008, 3:1.

73. Pawson R, Tilley N: Realistic evaluation. London: Sage; 1997.

74. Moher D, Hopewell S, Schulz KF, Montori V, Gøtzsche PC, Devereaux PJ, Elbourne DR, Egger M, Altman DG: CONSORT 2010 explanation and elaboration: updated guidelines for reporting parallel group randomised trials. BMJ 2010, 340:C869.

75. Zwarenstein M, Treweek S, Gagnier JJ, Altman DG, Tunis S, Haynes B, Oxman AD, Moher $\mathrm{D}$ : Improving the reporting of pragmatic trials: an extension of the CONSORT statement. BMJ 2008, 337:1223-1226.

76. Boutron I, Moher D, Altman DG, Schulz KF, Ravaud P: Extending the CONSORT statement to randomized trials of nonpharmacologic treatment: explanation and elaboration. Ann Intern Med 2008, 148:295-309.

77. Boutron I, Moher D, Altman DG, Schulz KF, Ravaud P: Methods and processes of the CONSORT group: example of an extension for trials assessing nonpharmacologic treatments. Ann Intern Med 2008, 148:W-60-W-66

78. Campbell MK, Elbourne DR, Altman DG: CONSORT statement: extension to cluster randomised trials. BMJ 2004, 328:702-708.

79. Brugler L, Stankovic A, Bernstein L, Scott F, O'Sullivan-Maillet J: The role of visceral protein markers in protein calorie malnutrition. Clin Chem Lab Med 2002, 40:1360-1369

doi:10.1186/1472-6963-13-299

Cite this article as: Kitson et al.: The prevention and reduction of weight loss in an acute tertiary care setting: protocol for a pragmatic stepped wedge randomised cluster trial (the PRoWL project). BMC Health Services Research 2013 13:299.

\section{Submit your next manuscript to BioMed Central and take full advantage of:}

- Convenient online submission

- Thorough peer review

- No space constraints or color figure charges

- Immediate publication on acceptance

- Inclusion in PubMed, CAS, Scopus and Google Scholar

- Research which is freely available for redistribution 